\title{
Peripheral mechanisms in tremor after traumatic neck injury
}

\author{
T Hashimoto, H Sato, M Shindo, R Hayashi, S Ikeda
}

J Neurol Neurosurg Psychiatry 2002;73:585-587

Tremor is a rare manifestation after neck injury, and its physiological mechanism has not been elucidated. We studied the effects of torque loading and ischaemic nerve block on coarse postural tremor in the right upper extremity, which had developed in association with a C7-C8 radiculopathy after traumatic neck injury in a 55 year old man. Loading reduced the tremor frequency from $6.1 \mathrm{~Hz}$ to $4.2 \mathrm{~Hz}$ with corresponding electromyography (EMG) bursts at the same frequencies as the tremor. Ischaemic nerve block also reduced the tremor frequency from 6.2 $\mathrm{Hz}$ to $2.8 \mathrm{~Hz}$, and the time course of the frequency was not in parallel with that of the size of the maximal $M$ wave. $A$ significant reduction of the tremor frequency by loading and ischaemic nerve block indicates a mechanical reflex mechanism underlying the tremor, and association of synchronous EMG bursts suggests an increase in gain in the stretch reflex loop. The stretch reflex loop plays an important role in generation of oscillation in tremor after neck injury.

$\mathrm{T}$ remor occasionally develops after peripheral injuries, ${ }^{1-3}$ but demonstration of central or peripheral aetiology in its oscillation generation is difficult. Modulation of tremor by mechanical perturbations or peripheral nerve shock may be not useful in differentiating a peripheral oscillator from a central one ${ }^{45}$ while analysis of load-frequency relationship ${ }^{1}$ or peripheral nerve block techniques ${ }^{6}$ have been applied to disclose involvement of peripheral nerves in tremor generation. We report a patient with medically intractable hand tremor developed after traumatic neck injury. We investigated contributions of peripheral components in the generation of the tremor by load application and ischaemic nerve block in the affected extremity.

\section{CASE REPORT}

A 55 year old man without a family history of tremor accidentally fell from a $4 \mathrm{~m}$ height to the ground. He had no blackout, but his neck and spine hit the ground. Seven days after the trauma, he developed a coarse postural tremor in the right upper extremity, along with numbness in the ulnar side of the right forearm. The hand tremor disabled him. Two years after the injury, he was referred to our department. Neurological examination revealed no abnormalities of the cranial nerves or cerebellar functions. Neck movements were not restricted and were painless. Sensation of light touch and pinprick was reduced in the right $\mathrm{C} 7$ and $\mathrm{C} 8$ distribution, and the right triceps brachii were slightly weak. Deep tendon reflexes and muscle tone were normal, plantar reflexes were negative, and no clonus was elicited in the extremities. The patient had a coarse postural tremor of about $6 \mathrm{~Hz}$ in the right arm and hand. The tremor was not present at rest. A fine postural tremor at $6-7 \mathrm{~Hz}$ by electromyography (EMG) examination was observed in the other limbs, but the tremor did not interfere motor performance. No other involuntary movement was noted. Needle EMG study revealed high amplitude motor units and polyphasic motor units in the triceps brachii and flexor carpi ulnaris muscles on the right side, suggesting damage to either the right C7 or C8 root. Brain MRI was normal, and cervical spine MRI showed a discal protrusion at C3-4, C6-7, and C7-Thl without cord compression. The patient's tremor was resistant to treatment with propranolol, arotinolol, diazepam, clonazepam, and carbamazepine.

\section{METHODS}

The patient gave informed consent for this investigation. In order to study the effects of loading on the tremor, he was seated with his right hand placed in a manipulandum coupled to a torque motor (Daiichi Keisoku, ADS-5, Japan), which was designed to restrict movement of the wrist joint in the horizontal plane. His hand was strapped to a moulded handle. A steady load was applied in a direction stretching the wrist extensor muscles, and the patient was asked to maintain a constant wrist position against the load. The torque, wrist angle, and surface EMG from the right forearm were recorded. The power spectra of the wrist angle and the full wave rectified and smoothed (upper cut off $15 \mathrm{~Hz}$ ) EMG were computed from 2.56 seconds of data with a resolution of $0.78 \mathrm{~Hz}$ in the frequency domain.

In order to study the effects of an ischaemic nerve block on the tremor, a tourniquet was placed on the right upper arm and inflated to $100 \mathrm{~mm} \mathrm{Hg}$ above the systolic blood pressure for 20 minutes. The patient put his right forearm on the platform with the palm down, and kept the wrist and fingers extended to produce constant tremor. Surface EMGs were recorded from the wrist flexor and extensor muscles. Tactile sensation and the size (area) of the maximal M wave (Mmax) from the wrist extensor muscles elicited by the radial nerve electrical stimulation delivered above the tourniquet were successively checked.

\section{RESULTS}

Without loading, the tremor frequency was $6.1 \mathrm{~Hz}$ and associated with EMG bursts of the same frequency. On loading of $0.78 \mathrm{Nm}$ in a direction of wrist flexion, the wrist angle showed 2-3 times increase in tremor amplitude, and EMG revealed reduction of the tremor frequency to $4.2 \mathrm{~Hz}$ with elongation of EMG burst duration of the wrist extensor (fig l).

In the nerve block study, the size of the Mmax began to decrease after eight minutes of ischaemia, and was reduced to $86 \%$ of control (the Mmax before ischaemia) after 18 minutes of ischaemia (fig 2A). Decrease in tactile sensation in the dorsum of the hand began after 10 minutes of ischaemia. The

Abbreviations: EMG, electromyography; RSD, reflex sympathetic dystrophy 
A

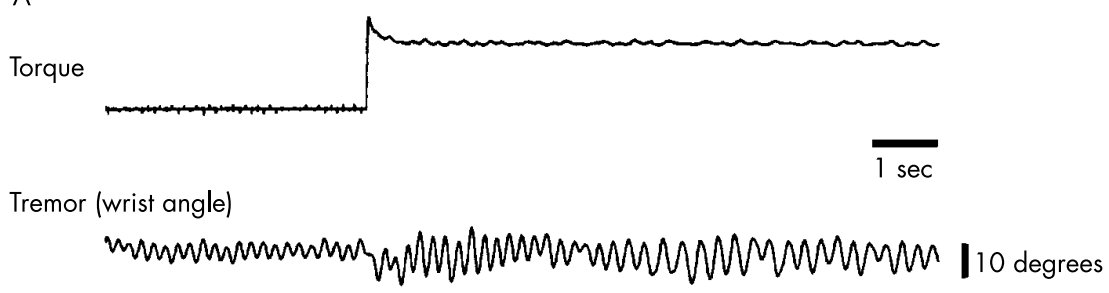

Wrist flexor EMG

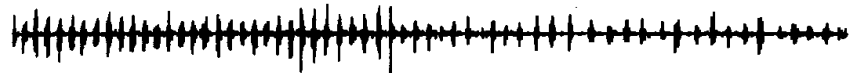

Wrist extensor EMG

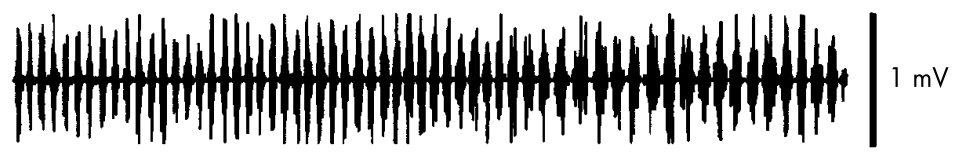

B

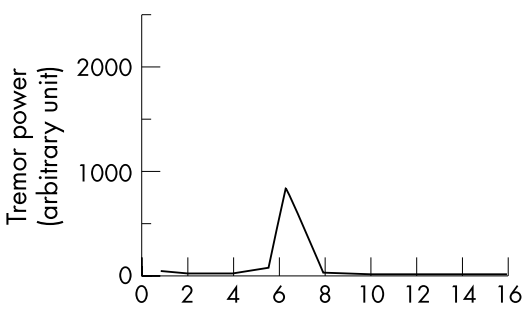

No load

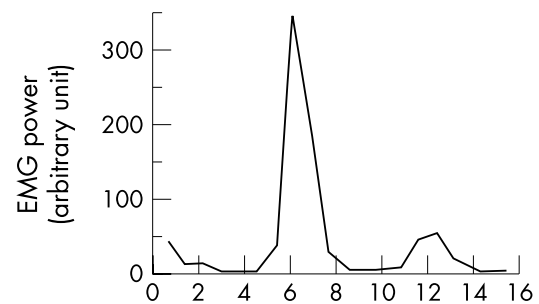

$0.78 \mathrm{Nm}$ load
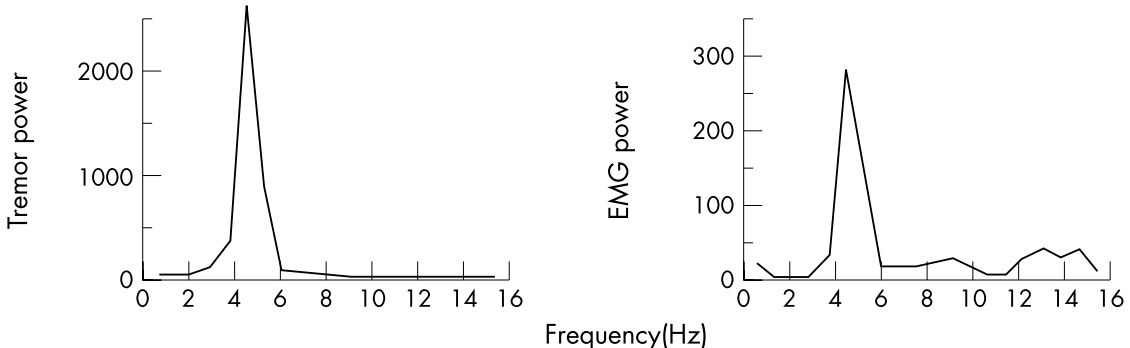

Figure 1 Effects of torque loading $(0.78 \mathrm{Nm})$ on the dorsum of the right hand in the direction of wrist flexion in the horizontal plane on the tremor. (A) Torque (top), wrist angle (second), and wrist flexor and extensor EMGs (third and bottom). (B) Spectra of tremor (wrist angle) and smoothed wrist extensor EMG. Without loading, the tremor spectrum showed a $6 \mathrm{~Hz}$ peak with a corresponding peak of the EMG spectrum at the same frequency. After loading, tremor frequency was reduced to about $4 \mathrm{~Hz}$ with corresponding reduction of EMG burst frequency.

tremor EMG discharges occurred at approximately $6.2 \mathrm{~Hz}$ before ischaemia. The tremor frequency gradually decreased with increased irregularity and reached a plateau at $2.8 \mathrm{~Hz}$ after 10 minutes of ischaemia (fig $2 \mathrm{~A}, \mathrm{~B}$ ). The patient could not maintain wrist extension after 18 minutes of ischaemia, and then the tremor disappeared. The tremor immediately resumed with recovery of voluntary wrist extension after deflation of the tourniquet.

\section{DISCUSSION}

The tremor of our patient developed a week after the fall in association with radiculopathy in the affected right upper extremity, indicating a causal relation to the trauma. ${ }^{2}$ Tremor developing after peripheral neck trauma has been reported, ${ }^{3}$ but the peripheral mechanisms underlying such tremor have not been determined. The existence of visible physiological tremor in unaffected limbs implies that the tremor in the right upper extremity may be activated physiological tremor ${ }^{78}$ enhanced by the injury. The association of postural tremor with reflex sympathetic dystrophy (RSD) has been reported, ${ }^{1}$ but our patient did not have autonomic symptoms, and the tremor frequency was lower than the range reported in RSD (mean $7.2($ SD 0.4$) \mathrm{Hz}){ }^{1}$

The tremor frequency in our patient was around $6 \mathrm{~Hz}$ and was associated with clear EMG bursts of the same frequency; the frequency was significantly (from $6.1 \mathrm{~Hz}$ to $4.2 \mathrm{~Hz}$ ) reduced by loading on the hand. Its association with EMG bursts indicates that the tremor is driven by a neuronal oscillator, ${ }^{9}$ while the reduction of tremor frequency by loading indicates the contribution of a mechanical reflex mechanism..$^{10}$ Tremors of central origin, parkinsonian tremor, or essential tremor are stable in frequency, irrespective of changes in load. ${ }^{8}$ The features observed in our patient are characteristics of activated physiological tremor, ${ }^{8}$ and the synchronisation of EMG bursts has been assumed to result from an increase in gain in the stretch reflex loop. ${ }^{8}$ Enhanced grouping of primary muscle spindle afferents has been 
A

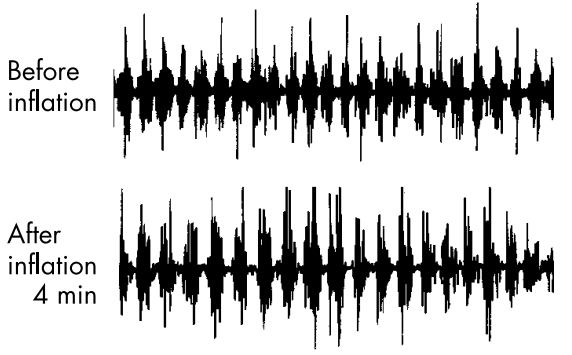

$10 \min$
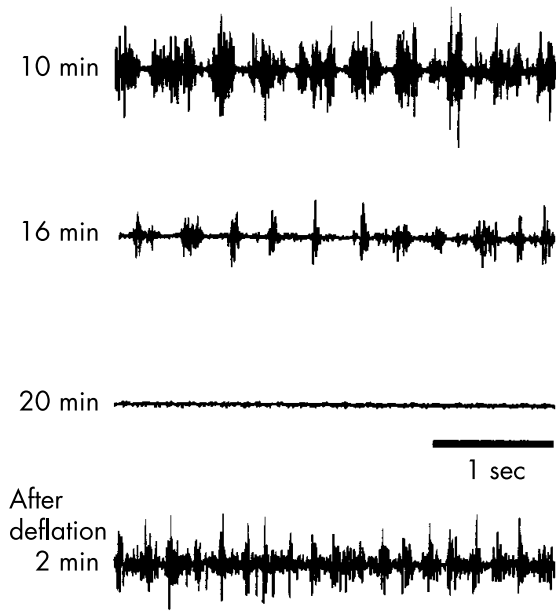

B

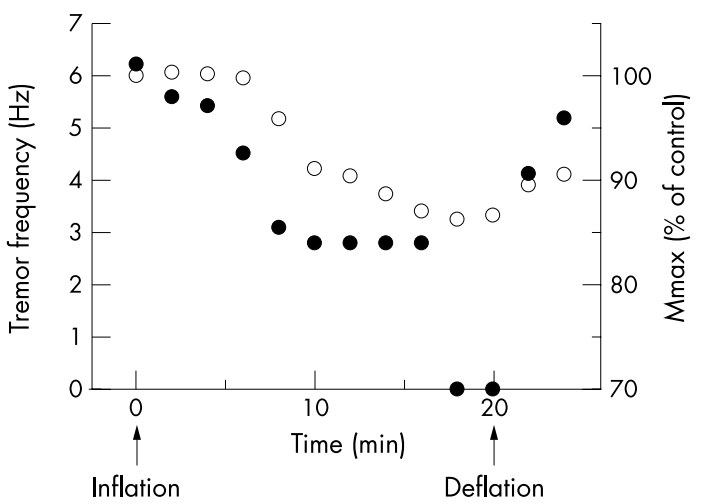

Figure 2 (A) Tremor EMGs recorded from the right wrist extensor muscles before, during, and after 20 minutes ischaemia at the upper arm. (B) Time course of the tremor frequency and the size of the Mmax expressed by \% of control (the Mmax before ischaemia). Tremor disappeared with difficulty in wrist extension after 18 minutes of ischemia. Open circles = size of $\mathrm{M}$ wave; filled circles $=$ tremor frequency.

observed in activated physiological tremor, and a sensitisation of spindle input has been assumed to underlie the increased gain. ${ }^{11}$

The reduction of tremor frequency during arm ischaemia in the patient reinforces the evidence indicating peripheral involvement in oscillation rhythm generation. Ischaemia blocks nerve conduction, depending on the size of the nerve fibres, and it also slows muscular contraction and neuromuscular transmission, ${ }^{12}$ not only by itself, but also by reduction of arm temperature. Therefore, reduction of tremor frequency during ischaemia can be caused by changes in mechanical property and also by changes in the activity of nerves. Close observation revealed that the decrease in tremor frequency during ischaemia started before the Mmax began to diminish, and the time courses of the tremor frequency and $\mathrm{M}$ wave were not in parallel in our patient, indicating that the tremor frequency depends on factors other than the number of conducting alpha motor axons. The muscle afferents may be more responsible for the initial reduction of tremor frequency. The plateau rhythm at $2.8 \mathrm{~Hz}$ after 10 minutes of ischaemia seems to be the lowest frequency to adjust the rhythm of synchronised motoneurone activity to mechanical resonance frequency.

The association of EMG bursts synchronous to the tremor and the reduction of tremor frequency by loading in our patient suggest peripheral tremogenic mechanisms similar to those in an activated physiological tremor. ${ }^{8}$ An increase in gain of the stretch reflex loop may underlie the tremor, but it remains unsolved whether the responsible reflex loop involves the central pathways or not, because altered peripheral input can produce central changes. ${ }^{2}$ Radiculopathy by itself is not associated with tremor in most cases, and participation of the neck injury in the peripheral alteration which causes the tremor remains obscure. Possible latent injury of the spinal cord may change the gain at the motoneurone level, or may change muscle spindle sensitivity.

\section{Authors' affiliations}

T Hashimoto, H Sato, S Ikeda, Third Department of Medicine, Shinshu University School of Medicine, Matsumoto, Japan

M Shindo, Shinshu University Center for Health Services, Matsumoto, Japan

R Hayashi, Department of Neurology, Okaya Municipal Hospital, Okaya, Japan

Competing interests: none declared

Correspondence to: Dr T Hashimoto, Third Department of Medicine, Shinshu University School of Medicine, Matsumoto, Japan; takahhh@hsp.md.shinshu-u.ac.jp

Received 25 March 2002

In revised form 31 July 2002

Accepted 8 August 2002

\section{REFERENCES}

1 Deuschl G, Blumberg $\mathrm{H}$, Lücking $\mathrm{CH}$. Tremor in reflex sympathetic dystrophy. Arch Neurol 1991;48:1247-52

2 Jankovic J. Post-traumatic movement disorders: central and peripheral mechanisms. Neurology 1994:44:2006-14.

3 Ellis SJ. Tremor and other movement disorders after whiplash type injuries. J Neurol Neurosurg Psychiatry 1997;63:1 10-12.

4 Britton TC, Thompson PD, Day BL, et al. "Resetting" of postural tremors at the wrist with mechanical stretches in Parkinson's disease, essential tremor, and normal subjects mimicking tremor. Ann Neurol 1992;31:507-14.

5 Britton TC, Thompson PD, Day BL, et al. Modulation of postural tremors at the wrist by supramaximal electrical median nerve shocks in essential tremor, Parkinson's disease and normal subjects mimicking tremor. J Neurol Neurosurg Psychiatry 1993;56:1085-9.

6 Lance JW, Schwab RS, Peterson EA. Action tremor and the cogwheel phenomenon in Parkinson's disease. Brain 1963:86:95-1 10.

7 Marsden CD, Obeso J, Rothwell JC. Benign essential tremor is not a single entity. In: Yahr MD, ed. Current concepts in Parkinson's disease. Amsterdam: Excerpta Medica, 1983:31-46.

8 Hömberg V, Hefter H, Reiners K, et al. Differential effects of changes in mechanical limb properties on physiological and pathological tremor. J Neurol Neurosurg Psychiatry 1987:50:568-79.

9 Elble RJ, Randall JE. Motor-unit activity responsible for 8 - to $12-\mathrm{Hz}$ component of human physiological finger tremor. J Neurophysiol 1976;39:370-83.

10 Stiles RN. Mechanical and neural feedback factors in postural hand tremor of normal subjects. J Neurophysiol 1980;44:40-59.

11 Hagbarth KE, Young RR. Participation of the stretch reflex in human physiological tremor. Brain 1979;102:509-26.

12 Moddel G, Best B, Ashby P. Effect of differential nerve block on inhibition of the monosynaptic reflex by vibration in man. J Neurol Neurosurg Psychiatry 1977;40:1066-71. 